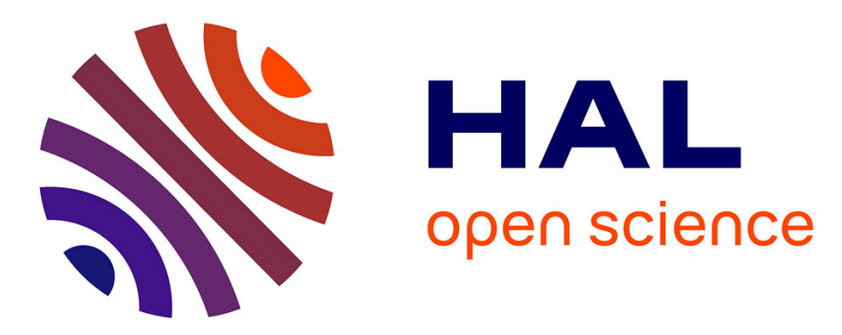

\title{
Controlled deposition and multi-layer architecturing of single biomolecules using automated directed capillary assembly and nano-contact printing processes
}

Hélène Cayron, Benjamin Berteloite, Christophe Vieu, Vincent Paveau, Jean Christophe Cau, Aline Cerf

\section{To cite this version:}

Hélène Cayron, Benjamin Berteloite, Christophe Vieu, Vincent Paveau, Jean Christophe Cau, et al.. Controlled deposition and multi-layer architecturing of single biomolecules using automated directed capillary assembly and nano-contact printing processes. Microelectronic Engineering, 2015, 135, pp. 1-6. 10.1016/j.mee.2015.02.011 . hal-01213795

\section{HAL Id: hal-01213795 \\ https://hal.science/hal-01213795}

Submitted on 9 Oct 2015

HAL is a multi-disciplinary open access archive for the deposit and dissemination of scientific research documents, whether they are published or not. The documents may come from teaching and research institutions in France or abroad, or from public or private research centers.
L'archive ouverte pluridisciplinaire HAL, est destinée au dépôt et à la diffusion de documents scientifiques de niveau recherche, publiés ou non, émanant des établissements d'enseignement et de recherche français ou étrangers, des laboratoires publics ou privés. 


\title{
Controlled deposition and multi-layer architecturing of single biomolecules using automated directed capillary assembly and nano-contact printing processes
}

\author{
Hélène Cayron ${ }^{1,2,4}$, Benjamin Berteloite ${ }^{4}$, Christophe Vieu ${ }^{1,2}$, Vincent Paveau ${ }^{4}$, Jean-Christophe Cau ${ }^{4}$, Aline Cerf ${ }^{1,3}$ \\ ${ }^{1}$ CNRS, LAAS, 7 avenue du colonel Roche, F-31400 Toulouse, France \\ ${ }^{2}$ Univ de Toulouse, INSA, LAAS, F-31400 Toulouse, France \\ ${ }^{3}$ Univ de Toulouse, LAAS, F-31400 Toulouse, France \\ ${ }^{4}$ Innopsys, Carbonne, 31390, France
}

\begin{abstract}
Up to date, a large amount of research studies have been carried out to manipulate and arrange biomolecules at the single molecule scale using capillary forces. However, many of these techniques remain in the fundamental research field, their industrial transfer being restricted by poor repeatability and user-dependent processes.

We present an automated process for the controlled and large scale deposition of single biomolecules, relying on the use of directed capillary assembly and nano-contact printing processes. The adjustment and control of physical parameters allow for single molecule deposition, and the use of an automate operating arm ensures high reproducibility and freedom in the assembly architectures to create.

This methodology was used to assemble DNA molecules and actin filaments (or F-actin), evidencing two distinct assembly mechanisms. In the first one we use capillary forces to trap and elongate preformed 1D structures such as DNA molecules. In the second case, fluid flows created upon evaporation and local pinning of the meniscus favor the F-actin polymerization through continuous supply of monomers. In this way, we introduce a new concept of using capillary assembly as a construction tool to assemble 1D nano-structures. Additionally, by sequentially aligning and printing multiple single molecule assemblies, large-scale multi-layer architectures of single molecules were also obtained.

The large-scale capabilities and reliability of our fabrication process render sophisticated single molecule biophysical measurements possible with systematic analysis over a large population for statistical relevance.
\end{abstract}

Keywords: Directed Capillary assembly - Nano-contact printing - DNA - F-actin

\section{Introduction}

In many single-molecule experiments, it is still a challenge to collect data on many molecules at once. For example, the pace of molecular motors research has often been limited by the low throughput of current methods for studying structural dynamics and mechanochemical activities in individual molecular machines. The controlled manipulation of single actin filaments (or F-actin) could thus open new possibilities for understanding how molecular motors, responsible for many key cellular processes, function. Another example concerns the fields of genetics and epigenetics. In the study of a variety of diseases, there is an active effort to map genome-wide genetic and epigenetic patterns across cell types. Interrogation of single elongated DNA or chromatin molecules could serve as a tool for medical diagnosis and monitoring of treatment effectiveness [1].

Since its first consideration by K. Nagayama et al [2], capillary assembly has been of growing interest in fields combining nano-patterning and biology in particular. Different approaches and configurations have been developed. The main idea relies on using capillary forces created when displacing a liquid containing micro/nano biological entities in suspension onto a substrate to efficiently trap and immobilize them, even uncoil and elongate them for 1D molecules [3]. By adjusting the surface tension of the fluid according to the substrate wettability properties and by patterning the substrate chemically or topographically, the deposition process can be controlled and the molecules arranged at definite positions onto the substrate [4]. Combining capillary assembly and nano-contact printing processes is a simple and low-cost process to arrange single biomolecules at the micrometric scale. The capability of directed capillary assembly to achieve single molecule deposition onto a micro-structured substrate, as well as their efficient transfer onto a target substrate by nanocontact printing stamp has already been demonstrated [5]. This process is a simple way to manipulate molecules without the need for clean room facilities, except for the fabrication of the silicon template or mold, which can then be re-used multiple times. The cost of raw materials can thus be very low, relying mostly on the use of polymeric stamps used as topographical substrates to guide the assembly of molecules. Nevertheless, a manual intervention is still required in most cases and accuracy in the printing position requires technical cunnings which often limit repeatability, thus restricting the transfer of this method to industrial processes. An automated tool would enable to organize biomolecules at a greater scale and ensure a process less user-dependent.

We have implemented a novel directed capillary assembly module in a commercial microcontact printer. Used in replacement to the conventional incubation to ink the stamp, capillary assembly allows a controlled inking of the latter and the controlled assembly of single biomolecules which can subsequently be transferred onto a final support via nano-contact printing in a completely automatized manner and with no user intervention. Two types of biomolecules were assembled to demonstrate the numerous capabilities and high versatility of our process: DNA molecules and F-actin. 


\section{Materials and methods}

\subsection{Preparation of the solutions, substrates and stamps}

DNA solution was prepared by diluting lambda phage DNA (Sigma, 48,502 bp, 329 $\mu \mathrm{g} / \mathrm{ml}$ ) in TBS buffer pH 8.0, at a concentration of $50 \mu \mathrm{g} / \mathrm{mL}$. After a $5 \mathrm{~min}$ incubation in a bath at $65^{\circ} \mathrm{C}$ followed by a $1 \mathrm{~min}$ ice-bath to separate molecular aggregates, labelling of the DNA double strands was achieved by adding 1.5 ul of $100 \mathrm{uM} \mathrm{YOYO-1}$ solution (Life Technologies) and letting the overall solution incubate overnight at $4^{\circ} \mathrm{C} .0 .5 \% \mathrm{v} / \mathrm{v}$ Triton $\mathrm{X}-100$ were then added right before starting the assembly process to adjust the contact angle of the solution $\left(23^{\circ} \pm 3^{\circ}\right.$ on polydimethylsiloxane (PDMS)). The final surface on which DNA molecules were transferred was functionalized with aminopropyltriethoxysilane (APTES) molecules (Sigma Aldrich, (3-Aminopropyl)triethoxysilane 99\%) for an optimal electrostatic immobilization of the DNA molecules. This coating is achieved by activating a glass slide with an air plasma (1min, $50 \mathrm{~W})$, then incubating the glass slide for 30min in $47.5 \mathrm{~mL}$ of ethanol, $2.5 \mathrm{~mL}$ of DI water and $500 \mu \mathrm{L}$ of APTES, and finally rinsing and heating the slide at $140^{\circ} \mathrm{C}$ for $5 \mathrm{~min}$.

F-actin was polymerized from a solution of monomeric globular actin (G-actin) at $1 \mathrm{mg} / \mathrm{mL}$ (Actin, from rabbit muscle Alexa Fluor ${ }^{\circledR} 488$ conjugate, in solution, Life Technologies).Using an actin polymerization buffer, the solution was incubated at room temperature for 30 minutes before adding $0.5 \% \mathrm{v} / \mathrm{v}$ of Triton X-100. Air plasma activation (1min, 50W) of the target glass slide is sufficient to immobilize actin filaments, provided that the process is achieved in the following hour.

Microstructured polydimethylsiloxane (PDMS) stamps were obtained by molding of PDMS (10:1 base to curing agent ratio) on a silicon etched mold hydrophobically treated, at $100^{\circ} \mathrm{C}$ for $1 \mathrm{~h}$. We used a generic stamp design compatible with the assembly of various molecules, consisting of $5 \mu \mathrm{m}$ in diameter cavities, organized according to a rectangular patterned area, with 9um periodicity along the $\mathrm{x}$ axis and 20um periodicity along the $\mathrm{y}$ axis. The total stamp area is $17 \times 14 \mathrm{~mm}$.

Characterization of the depositions was achieved using an inverted fluorescence microscope equipped with a 100X immersion objective, a fluorescence scanner (InnoScan1100®) and atomic force microscope (AFM) measurements with a Dimension Icon ${ }^{\circledR}$ AFM from Bruker using TESP-Super Sharp tips (Bruker, nominal radius 2nm), in tapping mode.

\subsection{Working principle of the micro-contact printing automate}

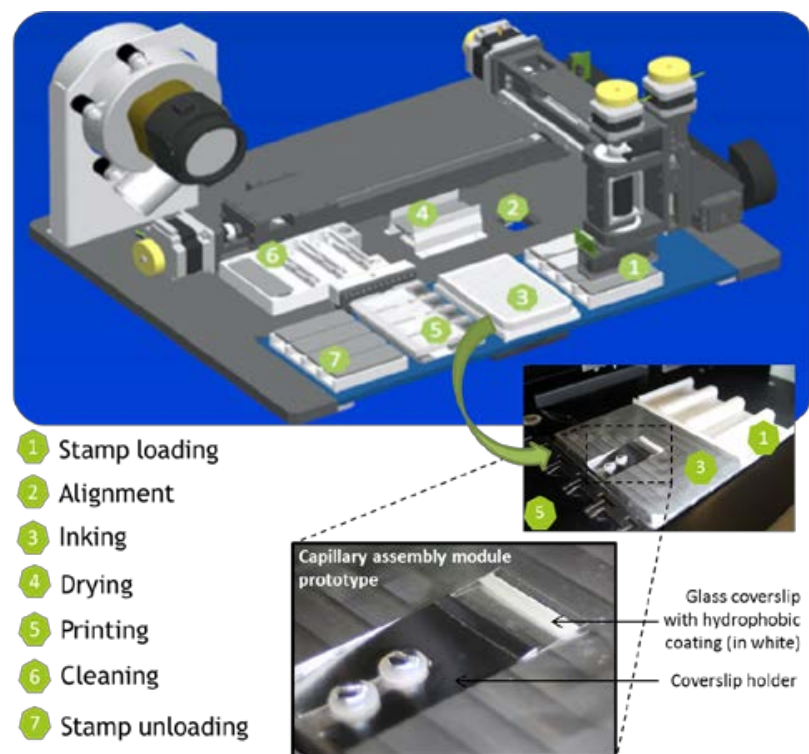

Figure 1: Process steps of the micro-contact printing automate. An inclined plane has been implemented in the inking zone for holding the glass coverslip to be used for capillary assembly (see section 2.3)

The InnoStamp ${ }^{\circledR}$ is a fully automated microcontact printer. It relies on the use of a magnetic field to easily manipulate a PDMS stamp magnetized on its upper part. The automate head containing magnets can pick up, move and release the stamp at will, in order to carry out all the routine steps of a standard micro-contact printing process, from loading of the stamp to inking, drying and printing (as seen in Fig 1). This microstructured stamp can be inked with the solution of interest via incubation of a droplet of the solution onto the stamp or by capillary assembly (see section 2.3) using the inclined plane implemented as seen in Fig.1. The stamp can then be dried if required, and placed in contact with a target surface to transfer the molecules via micro/nano-contact printing. During this step, the force applied on the stamp can be tuned through a magnetic field generated from the bottom of the substrate by $8 * 8$ magnets, ensuring a homogeneous printing process [6]. 


\subsection{Capillary assembly setup and process}

1



(2)

\section{$\sqrt{3}$}
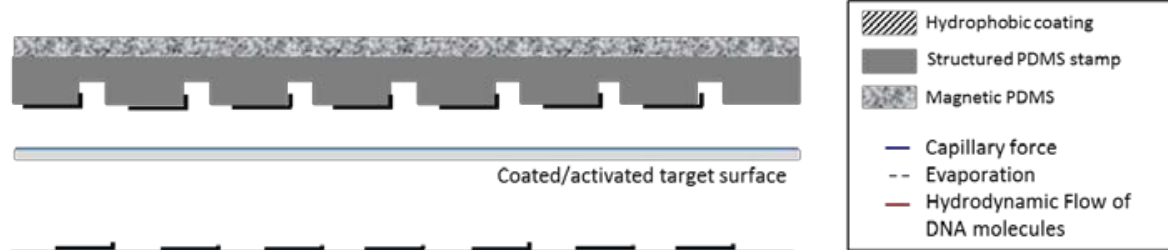

(3)

Coated/activated target surface

Figure 2: (1) Capillary assembly of molecules (case of DNA) in upside-down configuration. A glass coverslip with a hydrophobic coating at $5 \mathrm{~mm}$ from the edge to hold the droplet, is positioned with a $5^{\circ}$ angle from the horizontal plane. A side camera allows to precisely control the stamp-to-coverslip distance, $d$ (2) Nano-contact printing process: the molecules assembled onto the PDMS stamp are transferred to a target surface by simple contact with no additional force applied (3) Molecules assembled on the final surface.

Capillary assembly relies on the displacement of a droplet of solution containing the molecules of interest onto a structured substrate, here a PDMS structured stamp (see Fig 2). By adjusting the contact angle of the solution with a small amount of surfactant $(0.5 \% \mathrm{v} / \mathrm{v}$ of Triton X-100 was used in our case) and controlling the evaporation rate with displacement speed and temperature adjustment, a fluid flow is created inside the droplet with which the molecules are directed towards the contact line. When the liquid meniscus encounters the edge of a topographical structure, it is retained or pinned during a short lapse, which we will refer to as pinning time. The molecules concentrated at the triple line during that pinning time are subjected to the capillary forces exerted and trapped at the cavities of the stamp before the meniscus is finally released. These key experimental parameters have to be optimized and adapted depending on the molecule of interest in order to achieve a single molecule deposition

We implemented a capillary assembly module in the microcontact printing automate previously described. Compared to most of the setups commonly used for capillary assembly [7], here the assembly process is performed in an "upside-down" configuration, as seen in Fig.2, as part of an automatized microcontact printing global workflow with no user intervention.

The main asset of our setup is the presence of a side-camera which allows the experimenter to control the stamp-tocoverslip distance $d$ (see Fig 2 and annex 1 of the supplementary data), fixed at $0.3 \mathrm{~mm}$ in all our experiments, ensuring that the droplet only brushes the stamp in a repeatable manner and that the liquid layer at the meniscus is as thin as possible.

Once the assembly is performed, small droplets remain on the very edge of the stamp, corresponding to the end of assembly. These drops are removed using a blotting tissue, and nano-contact printing can be achieved. During this step, the stamp is placed in contact with the functionalized target surface (see Fig 2), and released with no force applied to preserve the molecules integrity before peeling the stamp off after one minute contact.

\section{Results and discussion}

\subsection{Large scale deposition of single DNA molecules}

DNA molecules, present in a coiled shape conformation in solution, can be elongated by the use of capillary forces; a process widely described and employed in previous references ([7], [8], [9]).

Previous development made with a less automated setup allowed to control the deposition of single DNA molecules (stamp displacement speed of $1 \mathrm{~mm} / \mathrm{s}$ and DNA concentration of $10 \mu \mathrm{g} / \mathrm{mL}$ ). Our setup configuration permits to control the stamp-to-coverslip distance $d$ (Fig 2.1). This characteristic distance $d$ has been observed to be a key parameter for the assemblies to be successful. In fact, the controlled squeezing of the droplet and the final height of the capillary bridge between the stamp and the coverslip induce an accumulation of molecules at the triple line favoring their assembly by capillarity. In our case and our present configuration, a concentration of $50 \mu \mathrm{g} / \mathrm{mL}$ was observed to be optimal to achieve a maximum occupation of the sites with single molecules. The stamp displacement speed was maintained at $1 \mathrm{~mm} / \mathrm{s}$ to induce the uncoiling or elongation of 1D molecules upon meniscus release. .

With a droplet of $40 \mu \mathrm{L}$ of solution, wide and homogeneous depositions were successfully achieved; with single elongated DNA molecules assembled over the entire $17 \mathrm{x} 14 \mathrm{~mm}^{2}$ surface of the PDMS stamp (see Fig 3c). 


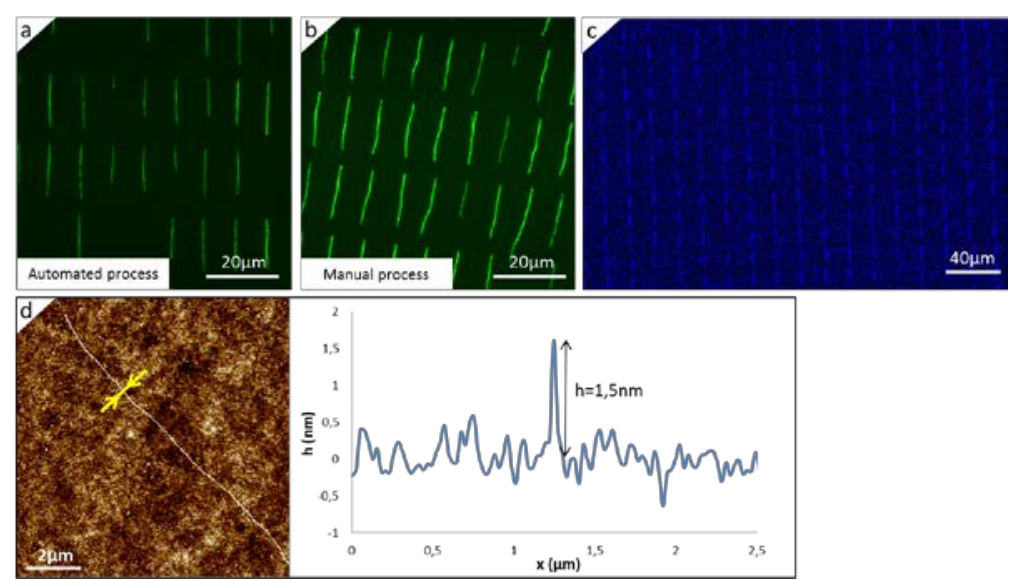

Figure 3: Fluorescence images of the deposition obtained with (a) our automated process with control of the distance d, and (b) a process as reported in [10]with no control in d and manual printing. A DNA solution at $50 \mu \mathrm{g} / \mathrm{mL}$ and a stamp-to-coverslip relative displacement of $1 \mathrm{~mm} / \mathrm{s}$ were used in both cases. (d)The AFM height measurements confirm the single molecule deposition in (a). (c) Large-scale characterization of single molecule deposition on the same sample (a) performed with a fluorescence scanner.

As seen in Fig. 3a and 3b, the use of a side camera in our automated process permits a better control of the assembly, favoring single molecule deposition at comparable DNA concentration. With the setup as reported in [10] with no control in $d$ and with manual printing, ropes of multiple molecules can be observed in various locations, and the inhomogeneous fluorescence signal from one site to the other highlights this multiple molecule deposition at that work concentration $(50 \mu \mathrm{g} / \mathrm{ml})$. In contrast, the same experiment conducted using the automated and upside-down setup with control in $d$ shows a more homogeneous and dim fluorescence signal where most of the sites are occupied by DNA molecules. These results were obtained repeatedly over various samples, with single DNA strands occupying $85 \%$ ( $\pm 5 \%)$ of the sites on average. The control in d ensures not having multiple molecule deposition at the chosen concentration of 50 $\mu \mathrm{g} / \mathrm{ml}$ in a repeatable manner. The AFM characterization confirms the single molecule deposition achieved with the automated process. The mean height measured perpendicularly to a molecule is $1.53 \mathrm{~nm}( \pm 0.31 \mathrm{~nm})$, close to the theoretical height of a DNA double strand of $2 \mathrm{~nm}$. The difference between the experimental and the theoretical values could be due to the fact that the molecules are transferred onto a layer of APTES molecules with a roughness of 260pm (Rq).

An area of the sample obtained was imaged with a fluorescence scanner (see Fig 3c). Despite of the high background noise due to glass inherent auto-fluorescence (this could be optimized using low-background commercial microscope supports), the scanner resolution is sufficient to discriminate the 220 DNA molecules regularly assembled in one field of view. Theoretically, we do not forecast any technical issue preventing us from assembling larger areas with a larger microstructured stamp.

DNA molecules were used as a demonstrator of the possibilities offered by this automated process. By controlling the meniscus thickness during capillary assembly and the transfer of the assembled molecules onto a target surface via nanocontact printing, one can obtain large-scale and well-organized deposition of single 1D molecules at the nano-scale. The most straightforward application of this process is the fabrication of ordered arrays of molecules to confer current DNA stretching techniques [8] with faster optical readout. Compared to conventional mapping in bulk material relying on the averaging of thousands of molecules, this technique allows mapping single molecules and could improve the understating of epigenetic modifications, at the single cell level.

Considering the latter, these studies could also be tremendously facilitated by knowing the exact locations of DNA molecules[11] or bead-on-a-string chromatin[12], allowing an automated and parallelized image acquisition and analysis of multiple molecules for genome and epigenome high-resolution mapping.

Knowing the exact locations of the molecules deposited with respect to the other could also drastically facilitate experiments relying on the manipulation of single molecules. Fundamental studies investigating DNA molecule properties, through the use of optical tweezers [13] or single Particle Tracking experiments [14] for example, could thus be pushed further by offering a parallelized, and more systematic and statistical approach.

\subsection{Creating architectures of single actin filaments}

F-actin assembly has to be considered otherwise than DNA molecule assembly, the formation of filaments taking place before but also during the assembly process. The comparison between the $1.5 \mathrm{um}$ on average filaments' lengths in "bulk" after 30min polymerization and the length obtained after capillary assembly which can reach $15 \mu \mathrm{m}$ with our design (see Fig 4a), evidenced a noteworthy increase in length due to the capillary assembly process. The polymerization process, still ongoing while the stamp displacement is initiated, may indeed be accelerated by the overall assembly physical process. First, the structuration of the stamp provokes the local anchoring of the meniscus at the sharp edges of the structures and each pinning point of the meniscus becomes a starting point for F-actin polymerization. Either independent monomers or short 
filaments already formed in solution are directed towards the contact line by the fluid flow created upon evaporation (see Fig 2.1), constantly supplying each local polymerization site of the stamp. This phenomenon thus drastically increases the polymerization rate of F-actin by increasing the pool of available monomers for polymerization, as compared to the probability of this occurring in three dimensions where diffusion is predominant.

As seen in Fig 4a, single actin filaments have been successfully polymerized in situ and simultaneously assembled resulting in regular arrays of single filaments of variable lengths across the substrate. Also, at the very start of each filament as indicated by the cross and arrow in Fig. 4a., the height seems increased compared to the mean height of the remaining portion. As seen in Fig. 4c, the height measured on the two first microns of an $8.5 \mu \mathrm{m}$ long filament is around $5.52 \mathrm{~nm}$ $( \pm 1.14 \mathrm{~nm})$, two times higher than the mean height measured elsewhere, $2.80 \mathrm{~nm}( \pm 0.66 \mathrm{~nm})$. This increased thickness could be the result of short filaments polymerized in solution and subjected to capillary forces during the meniscus pinning time, the polymerization being then favored for one of these filaments only, leading to a single filament formation. Another hypothesis could be that the surface wettability is locally modified as the polymerization occurs thus modifying the local pinning or deformability of the meniscus and the elastic vs pinning energies equilibrium. This would tend to over-concentrate monomers at the beginning of the pinning process and limit this phenomenon as the elastic energy stored exceeds the pinning energy finally releasing the meniscus and favoring single-filament formation. The mean height on the middle to end of the filament is indeed lower than the theoretical height of a single filament (from $6 \mathrm{~nm}$ to $8 \mathrm{~nm}$ ), which is comparable to previous measurements reported in literature [15].

For F-actin deposition, the sites filing rate with F-actin is close to $100 \%$. Monomers or short filaments are present in the solution at a high concentration and show a reduced spatial hindrance compared to long filaments or strands such as DNA molecules. The amount of actin monomers at the meniscus front line will thus be important, leading to a systematic trapping of a small aggregate of monomers acting as a germination center on each pattern. The repeatability in the filament length obtained from one filament to the other is then likely to depend on the evaporation-induced concentrating phenomenon at the front line [4].
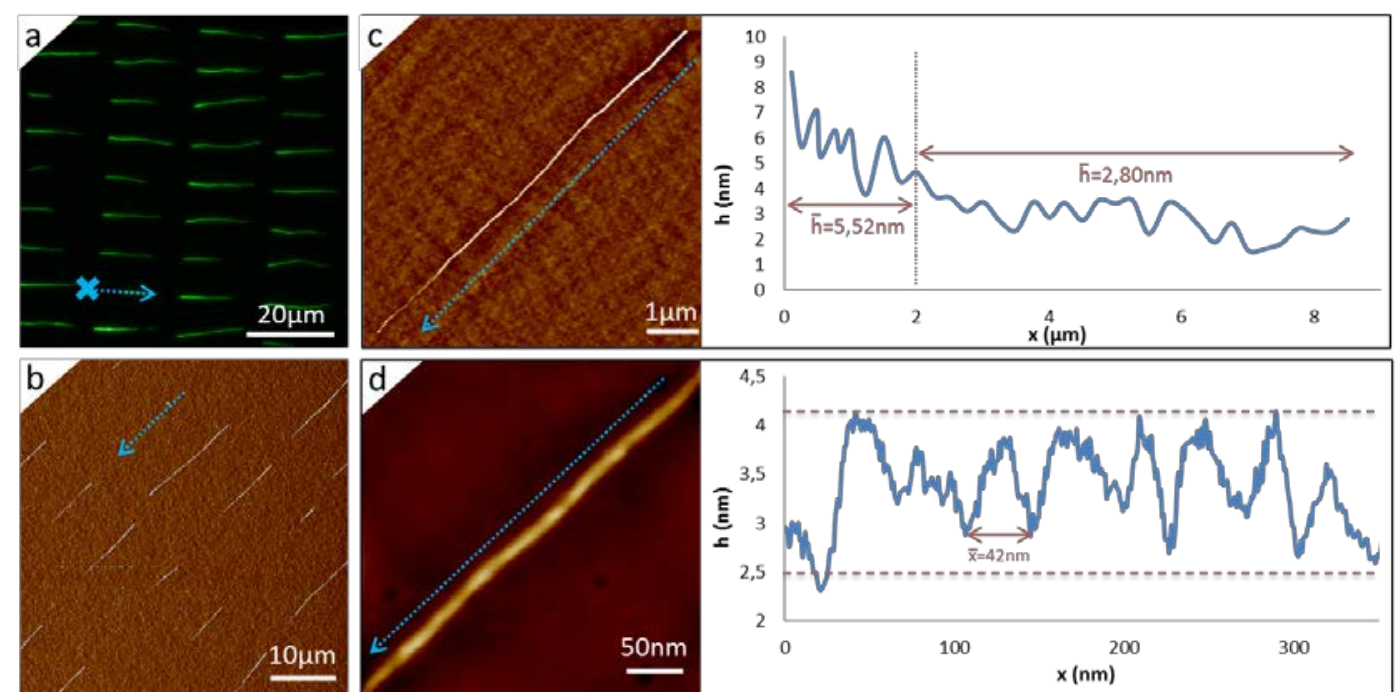

Figure 4: Fluorescence (a and annex 2 of the supplementary data) and AFM error (b) images of single actin filament deposition. Height measurements (c) from AFM corresponding height images confirm the presence of single actin filaments, and the preservation of F-actin helical structure is verified (d). The dotted blue arrows illustrate the assembly direction, and the blue cross in Fig. 4a. represents one positioning example of one of the PDMS cavities from which F-actin polymerization is initiated.

Assembling single filaments of such molecules can allow further investigation of their structure. The helical conformation of F-actin can indeed be evaluated by a height measurement along the filament, as seen in fig 4d. This measurement shows a repeated pattern every 42nm on average, characteristic of the helical structure pitch of F-actin and on the same order of magnitude of previous reports with a height oscillating between 2.7 and $4.2 \mathrm{~nm}$ on average [15].

The simple combination of assembly and printing of biomolecules can be considered and used as a repeatable and adjustable technological brick of a larger process, enabling the creation of multilayered molecular architectures. One can indeed achieve multiple assemblies and printing steps on the same target surface to arrange molecules according to a specific scheme, by choosing the right initial design for the structuration of the stamps. The molecules can also overlap between two layers. As a proof of principle, we achieved a "two-step" process, relying on the assembly and printing of single actin filaments in one direction, followed by a second assembly, printed on the same surface at a chosen angle from the first one (see Fig. 5). The process was achieved successfully, the second print being transferred as efficiently as the first one on the whole surface. 

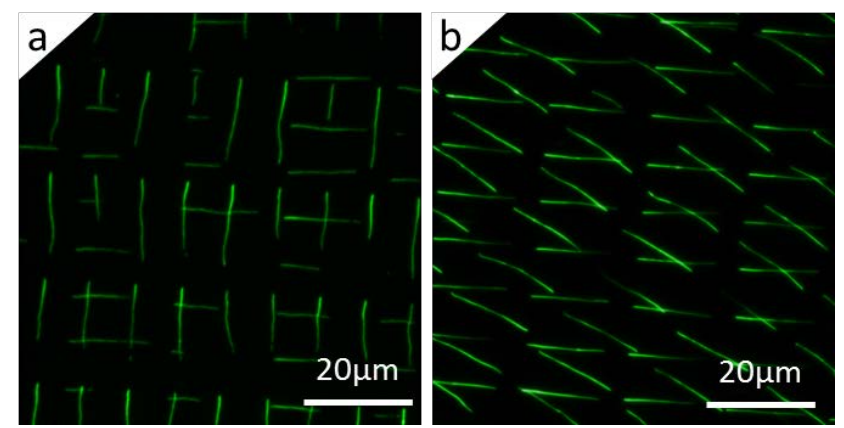

Figure 5: Fluorescence images of a two-step process with two consecutive prints of actin filaments (F-actin) on the same target surface, with a second print at $90^{\circ}$ (a) and $30^{\circ}$ angle (b) with respect to the first one

The versatility of this process does not only reside on the choice of molecules of interest and the initial design of the stamp, but also the target surface for printing these molecules. We currently envision printing onto a substrate equipped with trenches to obtain suspended 1D molecules for more dynamic biological studies. AFM force spectroscopy on single suspended DNA or chromatin molecules could allow investigating their physical properties such as their rigidity but also more complex dynamic physiological processes. We also have the ambition of using networks of suspended F-actin as pathways for myosin molecules to study their helical motion around complex actin architectures. Taking advantage of the automate positioning precision and designing the stamp structuration according to a specific scheme could allow the experimenter to create complex and specific networks with defined crossroad angles. The multi-step process could also be envisioned using different types of molecules for even more complex architecturing or layer-by-layer in vitro reconstitution of biological models.

\section{Conclusions}

We have automated the whole process of capillary assembly and printing of biomolecules at the single molecule level. This technique enables an increase in density, ordering and throughput capacity by generating a single-molecule platform in an automated way allowing massively parallel data collection in real time.

Our automated process showed the capability to generate single molecule deposition with high coverage rate and in a repeatable manner. Assembly and elongation of single 1D molecules in solution such as DNA molecules could be achieved. It was also shown that capillary assembly can favor and participate in the formation of $1 \mathrm{D}$ structures from isolated constituents which can self-assemble in situ, such as F-actin polymerization from G-actin monomers. Regular arrays of single actin filaments as confirmed by AFM could be obtained this way and for the first time to our knowledge. These immobilized molecules could be further characterized using super-resolution imaging techniques.

We have also shown the simplicity of creating nano-bio-architectures using this method. One major benefit of this process relies on its versatility in terms of choice of molecules, their final organization as determined by the stamp structuration design but also the choice of the final transfer surface. Future directions concern the generation of networks of suspended F-actin for the study of molecular motors helical motion or other dynamic physiological processes.

Many biomolecules are present as filaments in nature. We can thus imagine applying this process to other systems such as fibronectin [16], collagen [17]or desmin filaments[18] to generate patterned substrates for selective cell adhesion or interaction with extra cellular matrix components for example.

\section{Funding}

This work was achieved in the LAAS-CNRS/Innopsys joint laboratory BIOSOFT with the support of ANR-13-LAB2-000901

\section{References}

[1] A. Cerf, B. R. Cipriany, J. J. Benítez, and H. G. Craighead, "Single DNA Molecule Patterning for High-Throughput Epigenetic Mapping,” Anal. Chem., vol. 83, no. 21, pp. 8073-8077, Nov. 2011.

[2] P. . Kralchevsky, V. . Paunov, I. . Ivanov, and K. Nagayama, "Capillary meniscus interaction between colloidal particles attached to a liquid-fluid interface,” J. Colloid Interface Sci., vol. 151, no. 1, pp. 79-94, Jun. 1992.

[3] J. Guan and L. J. Lee, “Generating highly ordered DNA nanostrand arrays,” Proc. Natl. Acad. Sci., vol. 102, no. 51, pp. 18321-18325, Dec. 2005.

[4] L. Malaquin, T. Kraus, H. Schmid, E. Delamarche, and H. Wolf, "Controlled Particle Placement through Convective and Capillary Assembly,” Langmuir, vol. 23, no. 23, pp. 11513-11521, Nov. 2007. 
[5] A. Cerf, C. Thibault, M. Geneviève, and C. Vieu, "Ordered arrays of single DNA molecules by a combination of capillary assembly, molecular combing and soft-lithography,” Microelectron. Eng., vol. 86, no. 4-6, pp. 1419-1423, Apr. 2009.

[6] J.-C. Cau, L. Ludovic, N. Marie, L. Adriana, and P. Vincent, "Magnetic field assisted microcontact printing: A new concept of fully automated and calibrated process,” Microelectron. Eng., vol. 110, pp. 207-214, Oct. 2013.

[7] E. Palumbo, E. Tosoni, and A. Russo, "General and specific replication profiles are detected in normal human cells by genome-wide and single-locus molecular combing,” Exp. Cell Res., vol. 319, no. 20, pp. 3081-3093, Dec. 2013.

[8] J. N. Bianco, J. Poli, J. Saksouk, J. Bacal, M. J. Silva, K. Yoshida, Y.-L. Lin, H. Tourrière, A. Lengronne, and P. Pasero, "Analysis of DNA replication profiles in budding yeast and mammalian cells using DNA combing," Methods, vol. 57, no. 2, pp. 149-157, Jun. 2012.

[9] L. Cinque, A. Yamada, Y. Ghomchi, D. Baigl, and Y. Chen, “Cell trapping, DNA extraction and Molecular Combing in a microfluidic device for high throughput genetic analysis of human DNA,” Microelectron. Eng., vol. 88, no. 8, pp. 1733-1736, Aug. 2011.

[10] A. Cerf, X. Dollat, J. Chalmeau, A. Coutable, and C. Vieu, "A versatile method for generating single DNA molecule patterns: Through the combination of directed capillary assembly and (micro/nano) contact printing,” J. Mater. Res., vol. 26, no. 02, pp. 336-346, Jan. 2011.

[11] P. Pasero, "Single-molecule analysis reveals clustering and epigenetic regulation of replication origins at the yeast rDNA locus,” Genes Dev., vol. 16, no. 19, pp. 2479-2484, Oct. 2002.

[12] A. Cerf, H. C. Tian, and H. G. Craighead, "Ordered Arrays of Native Chromatin Molecules for High-Resolution Imaging and Analysis,” ACS Nano, vol. 6, no. 9, pp. 7928-7934, Sep. 2012.

[13] M. Spies, "Two steps forward, one step back: Determining XPD helicase mechanism by single-molecule fluorescence and high-resolution optical tweezers,” DNA Repair, vol. 20, pp. 58-70, Aug. 2014.

[14] T. PLENAT, L. SALOME, C. TARDIN, C. THIBAULT, E. TREVISIOL, and C. VIEU, "Biochips for analyzing nucleic acid molecule dynamics,” WO/2012/089946, 07-May-2012.

[15] T. Ikawa, F. Hoshino, O. Watanabe, Y. Li, P. Pincus, and C. Safinya, "Molecular Scale Imaging of F-Actin Assemblies Immobilized on a Photopolymer Surface,” Phys. Rev. Lett., vol. 98, no. 1, Jan. 2007.

[16] V. Nelea and M. T. Kaartinen, "Periodic beaded-filament assembly of fibronectin on negatively charged surface," $J$. Struct. Biol., vol. 170, no. 1, pp. 50-59, Apr. 2010.

[17] Y.-L. Sun, Z.-P. Luo, A. Fertala, and K.-N. An, “Stretching type II collagen with optical tweezers,” J. Biomech., vol. 37, no. 11, pp. 1665-1669, Nov. 2004.

[18] B. Kiss and M. S. Z. Kellermayer, "Stretching desmin filaments with receding meniscus reveals large axial tensile strength,” J. Struct. Biol., vol. 186, no. 3, pp. 472-480, Jun. 2014. 